\title{
Bi-space pullback attractors for closed processes
}

\section{Radosław Czaja}

Center for Mathematical Analysis, Geometry, and Dynamical Systems Departamento de Matemática, Instituto Superior Técnico

1049-001 Lisboa, Portugal

On the leave from: Institute of Mathematics, University of Silesia

Bankowa 14, 40-007 Katowice, Poland

E-mail address: czaja@math.ist.utl.pt

Dedicated to Luís Magalhães and Carlos Rocha on the occasion of their 60th birthdays

\begin{abstract}
In the description of the long-time behavior of solutions to nonautonomous differential equations the notion of a pullback attractor plays a similar role as the global attractor in autonomous dynamical systems. We present the theorem on the existence of a pullback attractor if the evolution process is a family of closed operators. The abstract result is formulated in the context of the smoothing properties of the process and for pullback attractors attracting a given universe, i.e. a chosen class of possibly time-dependent families of sets. We also present an application of the result to reaction-diffusion equations.
\end{abstract}

\section{Introduction}

Dynamical approach to autonomous differential equations leads to the construction of a semigroup and the asymptotic behavior of solutions involves questions about the global attractor. This approach brought various results on the existence of global attractors with most of the theorems requiring continuity of the semigroup (see e.g. [14], [26], [9]). However, this requirement may be sometimes difficult to obtain in the phase space, but

2000 Mathematics Subject Classification. Primary 37B55; Secondary 35B41.

Key words: Pullback attractor, nonautonomous dynamical systems, closed processes.

This work was initiated when the author visited Helmholtz Zentrum München. He thanks Messoud Efendiev and Anna Zhigun for their hospitality and fruitful discussions.

This research was partially supported by the FCT program Ciência 2008. 
some weaker continuity may still hold. One of the ways to release the assumption of continuity appears in [29], where semigroups satisfying normto-weak continuity are considered. This assumption can be substituted even by a weaker premise of closedness of operators forming the semigroup. This was done by Pata and Zelik in [20], where the authors proved existence of a global attractor for closed semigroups. Recently it has been shown in [10] that this can be obtained even for the class of asymptotically closed semigroups. Another advantage of the approach presented therein is consideration of bi-space global attractors, an idea already used e.g. in [2], [1], [8]. This allows more flexibility in proving compactness of the attractor and a broader basin of attraction for the semigroup. The above-mentioned ideas have been an inspiration for the present paper.

The nonautonomous differential equations became intensively investigated in the recent years. From the dynamical point of view this resulted in considering the analogue of a semigroup in the form of the evolution process. However, there is no unique equivalent notion for the global attractor - the uniform attractors, kernel sections, pullback attractors or forward attractors were explored among others (see [7], [6]).

First theorems on the existence of pullback attractors were formulated under the assumption of continuity of the process (see e.g. [11], [3]) and different assumptions of dissipativity and compactness. The continuity of the process is also a priori assumed in the monographs by Kloeden, Rasmussen [15] and Carvalho, Langa, Robinson [5]. The successful attempts to construct the pullback attractor without continuity were carried out e.g. in [16] and [17], where norm-to-weak (or strong-weak as in [19]) continuity of the process was used. This was done in the context of pullback $\mathcal{D}$-attractors in complete metric spaces and required that the process is dissipative possessing a family of pullback $\mathcal{D}$-absorbing sets and is pullback $\mathcal{D}$-limit set compact, which is a form of asymptotic compactness.

Recently appeared in the literature results concerning also closed processes. A similar result to the one from [20] was given in [25] in the case of a closed cocycle, which possesses a bounded absorbing set and the existing pullback attractor attracts bounded subsets of the phase space. Closed processes in the context of uniform attractors were also considered in [28].

In the present article we consider a process on a complete metric space $V$ and the universe $\mathcal{D}$ of families of nonempty subsets of $V$. We also assume that there exists a family of subsets of $V$, which pullback absorbs families from the universe. Moreover, we suppose that the absorbing family enters an auxiliary complete metric space $W$ in the pullback sense. The main Theorem 2.16 yields the existence of a pullback attractor with its sections compact in $W$ and attracting the universe $\mathcal{D}$ also in the topology of $W$. 
This is done under the assumptions of $(V-W)$ closedness (see Definition 2.12) and pullback asymptotic compactness. Additionally, we note in Corollary 2.17 that if the absorbing family $\hat{\mathcal{B}_{0}}$ belongs to the universe, it suffices to consider only the $(V-W)$ pullback $\left\{\hat{\mathcal{B}}_{0}\right\}$-asymptotic closedness of the process (see Definition 2.11).

During the preparation of this paper the author learned about the article [13], where the authors also explored the closed processes in more detail. Their Theorem 3.11 refers to the case of $V=W$ and the $(V-V)$ closed process.

Following [17] we illustrate the theory with an example of a reactiondiffusion equation in a bounded domain with Dirichlet boundary condition. Here the process is $\left(L^{2}(\Omega)-H_{0}^{1}(\Omega)\right)$ closed and the universe $\mathcal{D}$ is a class of suitable families of subsets of $L^{2}(\Omega)$, which contains all time-independent families of bounded subsets of $L^{2}(\Omega)$. The compactness of the sections of the pullback attractor as well as the attraction of $\mathcal{D}$ takes place in the space $H_{0}^{1}(\Omega)$.

\section{Closed processes and pullback attractors}

In this paper we consider evolution processes generated by nonautonomous differential equations.

Definition 2.1. Let $V$ be a nonempty set. A family $\{U(t, s): t \geq s\}$ of mappings $U(t, s): V \rightarrow V, t \geq s, t, s \in \mathbb{R}$, is called a process on $V$ if

$$
U(t, s)=U(t, \tau) U(\tau, s), t \geq \tau \geq s, \quad U(t, t)=I d_{V}, t \in \mathbb{R} .
$$

We pursue the investigation of the asymptotic behavior of these evolution processes by finding a pullback attractor for the process. In the genesis of the theory of pullback attractors (see [11]) the pullback attractor was a family of nonempty compact subsets of a metric space $V$ that was invariant under the process (see Definition 2.2(ii)) and attracted each bounded subset of $V$ (cp. Definition 2.2(iii)). This was later extended to the attraction of time-dependent families of (bounded) subsets of $V$. For the comparison of the two notions see [19]. Since we would like to have the generality as for bi-space global attractors (cp. [8]), we propose the following definition.

Let $\{U(t, s): t \geq s\}$ be a process on a complete metric space $\left(V, d_{V}\right)$ and let $\left(W, d_{W}\right)$ be an auxiliary complete metric space. Suppose also that $\mathcal{D}$ is a nonempty class of time-parameterized families $\hat{D}=\{D(t): t \in \mathbb{R}\}$ of nonempty subsets of $V$ such that

for any $\hat{D} \in \mathcal{D}$ and $t \in \mathbb{R}$ there exists $s_{\hat{D}, t} \leq t$ such that $\gamma_{s_{\hat{D}, t}}(\hat{D}, t) \subset W$, 
where we denote

$$
\gamma_{s_{0}}(\hat{D}, t)=\bigcup_{s \leq s_{0}} U(t, s) D(s) .
$$

The class $\mathcal{D}$ is sometimes called a universe (see e.g. [19]) and may require closedness with respect to inclusion (see [5, Definition 2.45], [15, Definition $3.23]$ ), which is not assumed here a priori.

Definition 2.2. The family $\hat{\mathcal{A}}=\{\mathcal{A}(t): t \in \mathbb{R}\}$ of subsets of $V$ is called a $(V-W)$ pullback $\mathcal{D}$-attractor if

(i) the set $\mathcal{A}(t) \subset V \cap W$ is nonempty and compact in $W$ for each $t \in \mathbb{R}$

(ii) $\hat{\mathcal{A}}$ is invariant under the process, i.e.,

$$
U(t, s) \mathcal{A}(s)=\mathcal{A}(t) \text { for } t \geq s,
$$

(iii) $\hat{\mathcal{A}}$ is pullback $\mathcal{D}$-attracting with respect to the Hausdorff semidistance dist $_{W}$ in $W$, i.e., for every $\hat{D} \in \mathcal{D}$ and $t \in \mathbb{R}$ we have

$\lim _{s \rightarrow-\infty} \operatorname{dist}_{W}(U(t, s) D(s), \mathcal{A}(t))=\lim _{s \rightarrow-\infty} \sup _{u \in D(s)} \inf _{v \in \mathcal{A}(t)} d_{W}(U(t, s) u, v)=0$,

(iv) $\hat{\mathcal{A}}$ is minimal in the sense that if another family $\hat{C}$ of nonempty closed subsets of $W$ pullback attracts $\mathcal{D}$, then $\hat{\mathcal{A}} \subset \hat{C}$, i.e., $\mathcal{A}(t) \subset$ $C(t), t \in \mathbb{R}$.

If $\mathcal{D}$ consists of all autonomous families of nonempty bounded subsets of $V$ and $V=W$, then the $(V-V)$ pullback $\mathcal{D}$-attractor coincides with the notion considered e.g. in [11].

The theorems on existence of global attractors or pullback attractors are based on certain dissipativity and compactness of the semigroup or process. Below we formulate two notions of compactness of a process. The first one follows e.g. [16, Definition 3.1] and uses the Kuratowski measure of noncompactness $\alpha_{W}$ in the space $W$

$\alpha_{W}(B)=\inf \{\delta>0: B$ has a finite cover by sets in $W$ of diameter $\leq \delta\}$,

where $B$ is a bounded subset of $W$ (see [22, Lemma 22.2] for its properties).

Definition 2.3. We say that the process $\{U(t, s): t \geq s\}$ is $(V-W)$ pullback $\mathcal{D}$-limit set compact if for any $\hat{D} \in \mathcal{D}, t \in \mathbb{R}$ and $\varepsilon>0$ there exists $t_{\hat{D}, t, \varepsilon} \leq s_{\hat{D}, t}$ such that

$$
\gamma_{t_{\hat{D}, t, \varepsilon}}(\hat{D}, t) \text { is bounded in } W \text { and } \alpha_{W}\left(\gamma_{t_{\hat{D}, t, \varepsilon}}(\hat{D}, t)\right)<\varepsilon \text {. }
$$


It is easy to see that if the process possesses a $(V-W)$ pullback $\mathcal{D}$ attractor, then it is necessarily $(V-W)$ pullback $\mathcal{D}$-limit set compact.

The other notion of compactness is based on [16, Definition 2.2] and is equivalent to the previous one (cp. [16, Theorem 3.3] and [27, Theorem $3.9])$

Definition 2.4. The process $\{U(t, s): t \geq s\}$ is $(V-W)$ pullback $\mathcal{D}$ asymptotically compact if for any $\hat{D} \in \mathcal{D}, t \in \mathbb{R}$ and any sequences $s_{n} \leq$ $s_{\hat{D}, t}, s_{n} \rightarrow-\infty$ and $x_{n} \in D\left(s_{n}\right) \subset V$, the sequence $\left\{U\left(t, s_{n}\right) x_{n}\right\}$ contains a convergent subsequence in $W$.

Proposition 2.5. The process $\{U(t, s): t \geq s\}$ is $(V-W)$ pullback $\mathcal{D}$-limit set compact if and only if the process $\{U(t, s): t \geq s\}$ is $(V-W)$ pullback $\mathcal{D}$-asymptotically compact.

The meaning of these two notions is that pullback $\omega$-limit sets $\omega_{W}(\hat{D}, t)$ in $W$ of families $\hat{D} \in \mathcal{D}$ are nonempty, compact in $W$ and pullback attract $\hat{D}$ at time $t$. By definition we set for $\hat{D} \in \mathcal{D}$ and $t \in \mathbb{R}$

$$
\omega_{W}(\hat{D}, t)=\bigcap_{s \leq s_{\hat{D}, t}} \operatorname{cl}_{W} \gamma_{s}(\hat{D}, t) .
$$

Equivalently, we have

$$
\begin{aligned}
& \omega_{W}(\hat{D}, t)= \\
& \quad\left\{w \in W: U\left(t, s_{n}\right) x_{n} \rightarrow w \text { in } W \text { for some } s_{n} \rightarrow-\infty \text { and } x_{n} \in D\left(s_{n}\right)\right\} .
\end{aligned}
$$

Proposition 2.6. The two conditions are equivalent:

(i) the process $\{U(t, s): t \geq s\}$ is $(V-W)$ pullback $\mathcal{D}$-asymptotically compact,

(ii) for any $\hat{D} \in \mathcal{D}$ and $t \in \mathbb{R}$ the set $\omega_{W}(\hat{D}, t)$ is nonempty, compact in $W$ and pullback attracts $\hat{D}$ at time $t$ w.r.t. dist $_{W}$.

Proof. The necessity of the condition (ii) follows along the lines of [4, Proposition 12] (cf. also [19, Proposition 2]). To see its sufficiency for the process to be $(V-W)$ pullback $\mathcal{D}$-asymptotically compact note that for fixed $\hat{D} \in \mathcal{D}, t \in \mathbb{R}, s_{n} \leq s_{\hat{D}, t}, s_{n} \rightarrow-\infty$ and $x_{n} \in D\left(s_{n}\right)$ and for any $k \in \mathbb{N}$ we can find $n_{k} \geq k$ and $w_{k} \in \omega_{W}(\hat{D}, t)$ such that $d_{W}\left(U\left(t, s_{n_{k}}\right) x_{n_{k}}, w_{k}\right)<1 / k$. By compactness of $\omega_{W}(\hat{D}, t)$ we choose a convergent subsequence of $\left\{w_{k}\right\}$. This gives a convergent subsequence of $\left\{U\left(t, s_{n}\right) x_{n}\right\}$ in $W$.

In order to guarantee $(V-W)$ pullback $\mathcal{D}$-asymptotic compactness in applications the following condition can be verified if $W$ is a Banach space. 
It had its origin in [18] and was later generalized to the case of processes (cp. [16, Definition 3.6]).

Definition 2.7. If $\left(W,\|\cdot\|_{W}\right)$ is a Banach space we say that the process $\{U(t, s): t \geq s\}$ on $V$ satisfies $(V-W)$ pullback $\mathcal{D}$-flattening condition if for any $\hat{D} \in \mathcal{D}, t \in \mathbb{R}$ and $\varepsilon>0$ there exist $t_{0}=t_{0}(\hat{D}, t, \varepsilon) \leq s_{\hat{D}, t}$ and a finite dimensional subspace $W_{1}=W_{1}(\hat{D}, t, \varepsilon)$ of $W$ together with a mapping $P=P(\hat{D}, t, \varepsilon): W \rightarrow W_{1}$ such that

(1) $M:=P\left(\gamma_{t_{0}}(\hat{D}, t)\right)$ is bounded in $W$,

(2) $R:=(I-P)\left(\gamma_{t_{0}}(\hat{D}, t)\right) \subset B^{W}(0, \varepsilon)$.

Proposition 2.8. If $\left(W,\|\cdot\|_{W}\right)$ is a Banach space and the process $\{U(t, s): t \geq s\}$ on $V$ satisfies $(V-W)$ pullback $\mathcal{D}$-flattening condition, then the process is $(V-W)$ pullback $\mathcal{D}$-limit set compact.

Proof. Let $\hat{D} \in \mathcal{D}, t \in \mathbb{R}$ and $\varepsilon>0$ and choose $t_{0}=t_{0}(\hat{D}, t, \varepsilon / 4) \leq s_{\hat{D}, t}$ and $W_{1}=W_{1}(\hat{D}, t, \varepsilon / 4)$ according to the assumption. Since $W_{1}$ is a finite dimensional subspace of $W$, it is a closed subspace of $W$. From (1) it follows that $\operatorname{cl}_{W} M=\operatorname{cl}_{W_{1}} M$ is bounded and closed in $W_{1}$, hence compact in $W_{1}$ (and $W$ ). Thus $M$ is precompact in $W$ and $\alpha_{W}(M)=0$. Moreover, $\gamma_{t_{0}}(\hat{D}, t)=M+R$ is bounded in $W$ and by $(2)$

$$
\alpha_{W}\left(\gamma_{t_{0}}(\hat{D}, t)\right) \leq \alpha_{W}(M)+\alpha_{W}(R)=\alpha_{W}(R) \leq \varepsilon / 2<\varepsilon .
$$

This shows that the process is $(V-W)$ pullback $\mathcal{D}$-limit set compact.

Remark 2.9. Observe that in order to obtain $(V-W)$ pullback $\mathcal{D}$-limit set compactness we could put in (2) a finite union of $\varepsilon$-balls in $W$ instead of $B^{W}(0, \varepsilon)$. Furthermore, we could use a more general splitting of the process $U(t, s)=S(t, s)+C(t, s), s \leq t_{0}$, instead of $U(t, s)=P U(t, s)+$ $(I-P) U(t, s)$.

The converse of Proposition 2.8 is also true in uniformly convex Banach spaces $W$ or, even more generally, in reflexive Banach spaces $W$, which are also strictly convex, i.e., where $\|x+y\|_{W}=\|x\|_{W}+\|y\|_{W}$ with $x, y \in$ $W \backslash\{0\}$ implies $y=c x$ for some $c>0$ (see [24, p. 35]). Equivalently, the strict convexity of $W$ means that for any $x, y \in W, x \neq y,\|x\|_{W}=$ $\|y\|_{W}=1$ and $\lambda \in(0,1)$ we have $\|\lambda x+(1-\lambda) y\|_{W}<1$. The strictly convex reflexive Banach spaces are characterized by the property that for any closed linear subspace $W_{1}$ and any $x \in W$ there exists a uniquely defined element $P x \in W_{1}$ such that $\|x-P x\|_{W}=\inf _{z \in W_{1}}\|x-z\|_{W}$ and thus the metric projection $P: W \rightarrow W_{1}$ is well-defined (see [12], [23, p. 111] 
or [24, Theorem 3.17]). Moreover, we have $\|P x\|_{W} \leq 2\|x\|_{W}, x \in W$, since $0 \in W_{1}$.

Proposition 2.10. If $\left(W,\|\cdot\|_{W}\right)$ is a reflexive strictly convex Banach space and the process $\{U(t, s): t \geq s\}$ on $V$ is $(V-W)$ pullback $\mathcal{D}$-limit set compact, then the process satisfies $(V-W)$ pullback $\mathcal{D}$-flattening condition.

Proof. Fix $\hat{D} \in \mathcal{D}, t \in \mathbb{R}$ and $\varepsilon>0$. By assumption there exists $t_{\hat{D}, t, \varepsilon} \leq s_{\hat{D}, t}$ such that $\gamma_{t_{\hat{D}, t, \varepsilon}}(\hat{D}, t)$ is bounded in $W$ and $\alpha_{W}\left(\gamma_{t_{\hat{D}, t, \varepsilon}}(\hat{D}, t)\right)<\varepsilon$. This means that there is a finite number $N$ of nonempty subsets $A_{1}, \ldots, A_{N}$ of $W$ with diameters less than $\varepsilon$ such that $\gamma_{t_{\hat{D}, t, \varepsilon}}(\hat{D}, t) \subset \bigcup_{i=1}^{N} A_{i}$. We choose $x_{i} \in A_{i}$ and set $W_{1}=\operatorname{span}\left\{x_{1}, \ldots, x_{N}\right\}$. By reflexivity and strict convexity of $W$ we know that there exists the metric projection $P: W \rightarrow W_{1}$ and $\|x-P x\|_{W}=\inf _{z \in W_{1}}\|x-z\|_{W}$ for each $x \in W$. The boundedness of $P$ implies that $P\left(\gamma_{\hat{D}, t, \varepsilon}(\hat{D}, t)\right)$ is bounded in $W$. Moreover, we get for $\begin{aligned} x \in \gamma_{t_{\hat{D}, t, \varepsilon}}(\hat{D}, t) & \\ \quad\|(I-P) x\|_{W} & =\inf _{z \in W_{1}}\|x-z\|_{W} \leq \inf _{z \in\left\{x_{1}, \ldots, x_{N}\right\}}\|x-z\|_{W}<\varepsilon .\end{aligned}$

This proves $(V-W)$ pullback $\mathcal{D}$-flattening condition.

Theorems on existence of global attractors require some kind of continuity argument for the semigroup. As already mentioned in the Introduction this can be reduced to the closedness of the semigroup (see [20]) or even to the asymptotic closedness (see [10]). Following the latter paper we give the next definition.

Definition 2.11. We say that the process $\{U(t, s): t \geq s\}$ on $V$ is $(V-W)$ pullback $\mathcal{D}$-asymptotically closed if for any $\hat{D} \in \mathcal{D}, t>s$ and $s_{n} \leq \min \left\{s_{\hat{D}, s}, s_{\hat{D}, t}\right\}, s_{n} \rightarrow-\infty$ and $x_{n} \in D\left(s_{n}\right)$

if $U\left(s, s_{n}\right) x_{n} \rightarrow v$ in $W$ and $U\left(t, s_{n}\right) x_{n} \rightarrow w$ in $W$, then $v \in V$ and $U(t, s) v=w$.

In some cases this may be insufficient, so we also define a stronger notion.

Definition 2.12. We say that the process $\{U(t, s): t \geq s\}$ on $V$ is $(V-W)$ closed if for any $t>s$ and $x_{n} \in V \cap W$ such that $U(t, s) x_{n} \in V \cap W$ if $x_{n} \rightarrow v$ in $W$ and $U(t, s) x_{n} \rightarrow w$ in $W$, then $v \in V$ and $U(t, s) v=w$.

The final ingredient of the results on existence of global attractors is dissipativity. 
Let $\{U(t, s): t \geq s\}$ be a process on a complete metric space $\left(V, d_{V}\right)$ and suppose now that $\mathcal{D}$ is a nonempty class of time-parameterized families $\hat{D}=\{D(t): t \in \mathbb{R}\}$ of nonempty subsets of $V$.

Definition 2.13. The process $\{U(t, s): t \geq s\}$ on $V$ is pullback $\mathcal{D}$-dissipative if there exists a pullback $\mathcal{D}$-absorbing family $\hat{\mathcal{B}}_{0}=\left\{\mathcal{B}_{0}(t): t \in \mathbb{R}\right\}$ of nonempty subsets of $V$, i.e., for any $\hat{D} \in \mathcal{D}$ and $t \in \mathbb{R}$ there exists $T_{\hat{D}, t} \leq t$ such that

$$
U(t, s) D(s) \subset \mathcal{B}_{0}(t), s \leq T_{\hat{D}, t} .
$$

The next proposition shows how the properties of $\hat{\mathcal{B}}_{0}$ translate into properties of $\mathcal{D}$.

Proposition 2.14. Let $\left(W, d_{W}\right)$ be a complete metric space. If $\hat{\mathcal{B}}_{0}=$ $\left\{\mathcal{B}_{0}(t): t \in \mathbb{R}\right\}$ is a pullback $\mathcal{D}$-absorbing family of nonempty subsets of $V$ for the process $\{U(t, s): t \geq s\}$ on $V$ such that

$$
\text { for any } t \in \mathbb{R} \text { there exists } s_{\hat{\mathcal{B}_{0}, t}} \leq t \text { such that } \gamma_{s_{\hat{\mathcal{B}}_{0}, t}}\left(\hat{\mathcal{B}_{0}}, t\right) \subset W \text {, }
$$

then (2.2) holds. Moreover, if the process is $(V-W)$ pullback $\left\{\hat{\mathcal{B}}_{0}\right\}$ asymptotically compact, then the process is $(V-W)$ pullback $\mathcal{D}$-asymptotically compact. Furthermore, if the process is $(V-W)$ pullback $\left\{\hat{\mathcal{B}}_{0}\right\}$-asymptotically closed, then it is $(V-W)$ pullback $\mathcal{D}$-asymptotically closed.

Proof. Fix $\hat{D} \in \mathcal{D}$ and $t \in \mathbb{R}$. By $(2.7)$ and (2.6) we set $s_{\hat{D}, t}:=T_{\hat{D}, s_{\hat{B_{0}}, t}} \leq$ $s_{\hat{\mathcal{B}}_{0}, t} \leq t$. Then for any $s \leq s_{\hat{D}, t}$ we have

$$
U(t, s) D(s)=U\left(t, s_{\hat{\mathcal{B}}_{0}, t}\right) U\left(s_{\hat{\mathcal{B}_{0}, t}}, s\right) D(s) \subset U\left(t, s_{\hat{\mathcal{B}_{0}}, t}\right) \mathcal{B}_{0}\left(s_{\hat{\mathcal{B}_{0}}, t}\right) \subset W,
$$

which gives (2.2). Moreover, if the process is $(V-W)$ pullback $\left\{\hat{\mathcal{B}}_{0}\right\}$ asymptotically compact, then for any sequences $s_{n} \leq \min \left\{s_{\hat{D}, t}, s_{\hat{\mathcal{B}}_{0}, t}\right\}$, $s_{n} \rightarrow-\infty$ and $x_{n} \in D\left(s_{n}\right)$ we choose a subsequence such that $s_{n_{k}} \leq$ $T_{\hat{D}, s_{k}} \leq s_{k}$ and have $U\left(t, s_{n_{k}}\right) D\left(s_{n_{k}}\right) \subset U\left(t, s_{k}\right) \mathcal{B}_{0}\left(s_{k}\right)$. Hence the sequence $\left\{U\left(t, s_{n_{k}}\right) x_{n_{k}}\right\}$ contains a convergent subsequence in $W$ and the process is $(V-W)$ pullback $\mathcal{D}$-asymptotically compact. The claim regarding $(V-W)$ pullback $\mathcal{D}$-asymptotic closedness follows by similar argument.

In the above proposition and Theorem 2.16 formulated below the properties for the class $\mathcal{D}$ follow from the properties of the family $\hat{\mathcal{B}_{0}}$ without assuming that $\hat{\mathcal{B}}_{0} \in \mathcal{D}$. Therefore we maintain the possibility that $\mathcal{D}$ consists of families of all time-independent bounded (or compact) sets.

Below we show that the pullback asymptotic closedness guarantees invariance of pullback $\omega$-limit sets under the process. 
Proposition 2.15. Let $\left(W, d_{W}\right)$ be a complete metric space and $\{U(t, s): t \geq s\}$ on $V$ be a pullback $\mathcal{D}$-dissipative process with $\hat{\mathcal{B}_{0}}$ being the pullback $\mathcal{D}$-absorbing family satisfying $(2.7)$. If the process is $(V-W)$ pullback $\left\{\hat{\mathcal{B}}_{0}\right\}$-asymptotically closed and $(V-W)$ pullback $\left\{\hat{\mathcal{B}}_{0}\right\}$-asymptotically compact, then $\omega_{W}(\hat{D}, t) \subset V$ for any $\hat{D} \in \mathcal{D} \cup\left\{\hat{\mathcal{B}}_{0}\right\}$ and $t \in \mathbb{R}$. Moreover, the family $\left\{\omega_{W}(\hat{D}, t): t \in \mathbb{R}\right\}$ is invariant under the process, i.e.

$$
U(t, s) \omega_{W}(\hat{D}, s)=\omega_{W}(\hat{D}, t), t \geq s
$$

Proof. By Proposition 2.14 the condition (2.2) holds and the process is $(V-W)$ pullback $\left(\mathcal{D} \cup\left\{\hat{\mathcal{B}}_{0}\right\}\right)$-asymptotically compact and closed. We fix $\hat{D} \in \mathcal{D} \cup\left\{\hat{\mathcal{B}}_{0}\right\}$ and $t>s$. Let $v \in \omega_{W}(\hat{D}, s)$. Then there exist $s_{n} \leq$ $\min \left\{s_{\hat{D}, s}, s_{\hat{D}, t}\right\}, s_{n} \rightarrow-\infty$ and $x_{n} \in D\left(s_{n}\right)$ such that $U\left(s, s_{n}\right) x_{n} \rightarrow \bar{v}$ in $W$. By $(V-W)$ pullback $\{\hat{D}\}$-asymptotic compactness there exists a subsequence $\left\{n_{k}\right\}$ and $w \in \omega_{W}(\hat{D}, t)$ such that $U(t, s) U\left(s, s_{n_{k}}\right) x_{n_{k}} \rightarrow$ $w$ in $W$. By $(V-W)$ pullback $\{\hat{D}\}$-asymptotic closedness we get $v \in$ $V$ and $U(t, s) v=w \in \omega_{W}(\hat{D}, t)$. This proves that $\omega_{W}(\hat{D}, s) \subset V$ and $U(t, s) \omega_{W}(\hat{D}, s) \subset \omega_{W}(\hat{D}, t)$.

Let now $w \in \omega_{W}(\hat{D}, t)$. Then there exist $s_{n} \leq \min \left\{s_{\hat{D}, t}, s_{\hat{D}, s}\right\}, s_{n} \rightarrow-\infty$ and $x_{n} \in D\left(s_{n}\right)$ such that $U\left(t, s_{n}\right) x_{n}=U(t, s) U\left(s, s_{n}\right) x_{n} \rightarrow w$ in $W$. By $(V-W)$ pullback $\{\hat{D}\}$-asymptotic compactness there exists a subsequence $\left\{n_{k}\right\}$ and $v \in \omega_{W}(\hat{D}, s)$ such that $U\left(s, s_{n_{k}}\right) x_{n_{k}} \rightarrow v$ in $W$. Thus $(V-W)$ pullback $\{\hat{D}\}$-asymptotic closedness implies $v \in V$ and $U(t, s) v=w$ and $\omega_{W}(\hat{D}, t) \subset U(t, s) \omega_{W}(\hat{D}, s)$.

Below we present the main result of this section on the existence of bispace pullback attractors for closed processes.

Theorem 2.16. Let $\{U(t, s): t \geq s\}$ be a process on a complete metric space $V$ and let $W$ be an auxiliary complete metric space. Suppose also that $\mathcal{D}$ is a nonempty class of time-parameterized families $\hat{D}=\{D(t): t \in \mathbb{R}\}$ of nonempty subsets of $V$. Assume that the process is pullback $\mathcal{D}$-dissipative with $\hat{\mathcal{B}_{0}}$ being the pullback $\mathcal{D}$-absorbing family in $V$ satisfying (2.7). If the process is $(V-W)$ closed and $(V-W)$ pullback $\left\{\hat{\mathcal{B}}_{0}\right\}$-asymptotically compact, then there exists a $(V-W)$ pullback $\mathcal{D}$-attractor $\hat{\mathcal{A}}=\{\mathcal{A}(t): t \in$ $\mathbb{R}\}$ for the process $\{U(t, s): t \geq s\}$ given by

$$
\mathcal{A}(t)=\operatorname{cl}_{W} \bigcup_{\hat{D} \in \mathcal{D}} \omega_{W}(\hat{D}, t) \subset \omega_{W}\left(\hat{\mathcal{B}}_{0}, t\right), t \in \mathbb{R} .
$$


Proof. From Proposition 2.14 it follows that the process $\{U(t, s): t \geq s\}$ is $(V-W)$ pullback $\left(\mathcal{D} \cup\left\{\hat{\mathcal{B}}_{0}\right\}\right)$-asymptotically compact. By Proposition 2.6, for any $\hat{D} \in \mathcal{D} \cup\left\{\hat{\mathcal{B}}_{0}\right\}$ and any $t \in \mathbb{R}$ the set $\omega_{W}(\hat{D}, t)$ is nonempty, compact in $W$ and pullback attracts $\hat{D}$ at time $t$ w.r.t. dist $_{W}$. We define

$$
\mathcal{A}(t):=\operatorname{cl}_{W} \bigcup_{\hat{D} \in \mathcal{D}} \omega_{W}(\hat{D}, t), t \in \mathbb{R} .
$$

Since $\mathcal{D}$ is nonempty, $\mathcal{A}(t)$ is also nonempty. By definition it is a closed subset of $W$ and pullback attracts each $\hat{D} \in \mathcal{D}$ at time $t \in \mathbb{R}$ w.r.t. $\operatorname{dist}_{W}$. To verify the minimality property (Definition $2.2($ iv)), let $\{C(t): t \in \mathbb{R}\}$ be a family of nonempty closed subsets of $W$ such that for $t \in \mathbb{R}$ and $\hat{D} \in \mathcal{D}$ we know that $\operatorname{dist}_{W}(U(t, s) D(s), C(t)) \rightarrow 0$ as $s \rightarrow-\infty$. Let $w \in \omega_{W}(\hat{D}, t)$. Then there exist $s_{n} \leq s_{\hat{D}, t}, s_{n} \rightarrow-\infty$ and $x_{n} \in D\left(s_{n}\right)$ such that $U\left(t, s_{n}\right) x_{n} \rightarrow w$ in $W$. Thus for any $k \in \mathbb{N}$ there are $n_{k} \in \mathbb{N}$ and $z_{k} \in C(t)$ such that $d_{W}\left(U\left(t, s_{n_{k}}\right) x_{n_{k}}, z_{k}\right)<1 / k$. This implies $z_{k} \rightarrow w$ in $W$ and $w \in \operatorname{cl}_{W} C(t)=C(t)$ and hence we obtain $\mathcal{A}(t) \subset C(t)$.

Observe that for any $\hat{D} \in \mathcal{D}$ and $t \in \mathbb{R}$ we have

$$
\lim _{s \rightarrow-\infty} \operatorname{dist}_{W}\left(U(t, s) D(s), \omega_{W}\left(\hat{\mathcal{B}_{0}}, t\right)\right)=0 .
$$

Indeed, for $\varepsilon>0$ fixed, let $t_{\hat{\mathcal{B}_{0}, t, \varepsilon}} \leq s_{\hat{\mathcal{B}}_{0}, t}$ be such that $\operatorname{dist}_{W}\left(U(t, s) \mathcal{B}_{0}(s), \omega_{W}\left(\hat{\mathcal{B}_{0}}, t\right)\right)<\varepsilon$ for $s \leq t_{\hat{\mathcal{B}}_{0}, t, \varepsilon}$. Moreover, from (2.6) there exists $T_{\hat{D}, t_{\hat{\mathcal{B}}_{0}, t, \varepsilon}} \leq t_{\hat{\mathcal{B}}_{0}, t, \varepsilon}$ such that we have $U\left(t_{\hat{\mathcal{B}}_{0}, t, \varepsilon}, s\right) D(s) \subset \mathcal{B}_{0}\left(t_{\hat{\mathcal{B}}_{0}, t, \varepsilon}\right)$ for $s \leq T_{\hat{D}, t_{\hat{\mathcal{B}}_{0}, t, \varepsilon}}$. Thus we obtain for $s \leq T_{\hat{D}, t_{\hat{\mathcal{B}}_{0}, t, \varepsilon}}$

$\operatorname{dist}_{W}\left(U(t, s) D(s), \omega_{W}\left(\hat{\mathcal{B}_{0}}, t\right)\right) \leq \operatorname{dist}_{W}\left(U\left(t, t_{\hat{\mathcal{B}}_{0}, t, \varepsilon}\right) \mathcal{B}_{0}\left(t_{\hat{\mathcal{B}}_{0}, t, \varepsilon}\right), \omega_{W}\left(\hat{\mathcal{B}_{0}}, t\right)\right)<\varepsilon$

Concluding, $\left\{\omega_{W}\left(\hat{\mathcal{B}}_{0}, t\right): t \in \mathbb{R}\right\}$ is a family of nonempty, compact subsets of $W$, pullback attracting $\mathcal{D}$ w.r.t. dist ${ }_{W}$. By the above considerations it follows that for any $\hat{D} \in \mathcal{D}$ and $t \in \mathbb{R}$ we have $\omega_{W}(\hat{D}, t) \subset \omega_{W}\left(\hat{\mathcal{B}}_{0}, t\right)$. This implies that $\mathcal{A}(t) \subset \omega_{W}\left(\hat{\mathcal{B}}_{0}, t\right), t \in \mathbb{R}$, and $\mathcal{A}(t)$ is a compact subset of $W$.

Note that using only $(V-W)$ pullback $\left\{\hat{\mathcal{B}}_{0}\right\}$-asymptotic closedness of the process, it follows from Proposition 2.15 that $\mathcal{A}(t) \subset \omega_{W}\left(\hat{\mathcal{B}_{0}}, t\right) \subset V$ for $t \in \mathbb{R}$ and for any $\hat{D} \in \mathcal{D} \cup\left\{\hat{\mathcal{B}}_{0}\right\}$ and $t \geq s$ we have $U(t, s) \omega_{W}(\hat{D}, s)=$ $\omega_{W}(\hat{D}, t)$.

If $\hat{\mathcal{B}}_{0} \notin \mathcal{D}$, we now use the stronger assumption of $(V-W)$ closedness of the process to prove the invariance of the family $\{\mathcal{A}(t): t \in \mathbb{R}\}$. 
Indeed, let $t>s$ and $v \in \mathcal{A}(s)$. Then there exist $x_{n} \in \omega_{W}\left(\hat{D}_{n}, s\right) \subset$ $V \cap W$ for some $\hat{D}_{n} \in \mathcal{D}$ such that $x_{n} \rightarrow v$ in $W$. We know that $U(t, s) x_{n} \in$ $\omega_{W}\left(\hat{D}_{n}, t\right) \subset \mathcal{A}(t) \subset V \cap W$. By compactness in $W$ of $\mathcal{A}(t)$ there exists a subsequence $\left\{n_{k}\right\}$ and $w \in \mathcal{A}(t)$ such that $U(t, s) x_{n_{k}} \rightarrow w$ in $W$. By the $(V-W)$ closedness of the process we get $U(t, s) v=w$ and $U(t, s) \mathcal{A}(s) \subset$ $\mathcal{A}(t)$.

On the other hand, if $w \in \mathcal{A}(t)$ then there exist $y_{n} \in \omega_{W}\left(\hat{D}_{n}, t\right) \subset V \cap W$ for some $\hat{D}_{n} \in \mathcal{D}$ such that $y_{n} \rightarrow w$ in $W$. We know that there are $x_{n} \in \omega\left(\hat{D}_{n}, s\right) \subset \mathcal{A}(s) \subset V \cap W$ such that $U(t, s) x_{n}=y_{n}$. By compactness in $W$ of $\mathcal{A}(s)$ there exists a subsequence $\left\{n_{k}\right\}$ such that $x_{n_{k}} \rightarrow v \in \mathcal{A}(s) \subset$ $V \cap W$ in $W$. By $(V-W)$ closedness of the process $U(t, s) v=w$ and $\mathcal{A}(t) \subset U(t, s) \mathcal{A}(s)$. This ends the proof.

From the proof of the above theorem we get the direct generalization to evolution processes of Theorem 4.1 from [10] .

Corollary 2.17. If we substitute in Theorem 2.16 the $(V-W)$ closedness of the process $\{U(t, s): t \geq s\}$ by a weaker requirement that the process is $(V-W)$ pullback $\left\{\hat{\mathcal{B}}_{0}\right\}$-asymptotically closed and assume additionally that $\hat{\mathcal{B}_{0}} \in \mathcal{D}$, then there exists a $(V-W)$ pullback $\mathcal{D}$-attractor $\hat{\mathcal{A}}=\{\mathcal{A}(t): t \in \mathbb{R}\}$ for the process $\{U(t, s): t \geq s\}$ and is given by $\mathcal{A}(t)=\omega_{W}\left(\hat{\mathcal{B}_{0}}, t\right), t \in \mathbb{R}$.

According to Proposition 2.5, in Theorem 2.16 and Corollary 2.17 the $(V-W)$ pullback $\left\{\hat{\mathcal{B}}_{0}\right\}$-asymptotic compactness is equivalent to the $(V-W)$ pullback $\left\{\hat{\mathcal{B}}_{0}\right\}$-limit set compactness of the process and, by Proposition 2.8, can be substituted by the $(V-W)$ pullback $\mathcal{D}$-flattening condition if $W$ is a Banach space.

Note that if $\hat{\mathcal{B}}_{0}$ is a family of bounded subsets of $V$ which really depend on time $t$, then in the above corollary we cannot take in the role of $\mathcal{D}$ the class consisting solely of all constant time-independent families of nonempty bounded subsets of $V$.

The condition of $(V-W)$ pullback $\left\{\hat{\mathcal{B}}_{0}\right\}$-limit set compactness of the process can be expressed differently if we know that $\hat{\mathcal{B}}_{0}$ also absorbs itself. This together with the above considerations gives a direct generalization of [20, Theorem 2].

Proposition 2.18. Let $\{U(t, s): t \geq s\}$ be a process on a metric space $V$ and let $W$ be an auxiliary complete metric space. Assume that $\hat{\mathcal{B}}_{0}=$ $\left\{\mathcal{B}_{0}(t): t \in \mathbb{R}\right\}$ is a family of nonempty subsets of $V$ satisfying (2.7) such that for any $t \in \mathbb{R}$ there exists $T_{\hat{\mathcal{B}}_{0}, t} \leq t$ such that

$$
U(t, s) \mathcal{B}_{0}(s) \subset \mathcal{B}_{0}(t), s \leq T_{\hat{\mathcal{B}}_{0}, t} .
$$


The process is $(V-W)$ pullback $\left\{\hat{\mathcal{B}}_{0}\right\}$-limit set compact if and only if for each $t \in \mathbb{R}$ and $\varepsilon>0$ there exists $t_{\hat{\mathcal{B}}_{0}, t, \varepsilon} \leq s_{\hat{\mathcal{B}_{0}, t}}$ such that

$U\left(t, t_{\hat{\mathcal{B}_{0}, t, \varepsilon}}\right) \mathcal{B}_{0}\left(t_{\hat{\mathcal{B}_{0}}, t, \varepsilon}\right)$ is bounded in $W$ and $\alpha_{W}\left(U\left(t, t_{\hat{\mathcal{B}_{0}, t, \varepsilon}}\right) \mathcal{B}_{0}\left(t_{\hat{\mathcal{B}_{0}, t, \varepsilon}}\right)\right)<\varepsilon$.

Proof. To show that the above condition is sufficient note that for $t \in \mathbb{R}$ and $\varepsilon>0$ fixed and for $s \leq T_{\hat{\mathcal{B}}_{0}, t_{\hat{\mathcal{B}_{0}}, t, \varepsilon}} \leq t_{\hat{\mathcal{B}_{0}}, t, \varepsilon}$ we have by $(2.8)$

$$
U(t, s) \mathcal{B}_{0}(s)=U\left(t, t_{\hat{\mathcal{B}_{0}, t, \varepsilon}}\right) U\left(t_{\hat{\mathcal{B}}_{0}, t, \varepsilon}, s\right) \mathcal{B}_{0}(s) \subset U\left(t, t_{\hat{\mathcal{B}_{0}}, t, \varepsilon}\right) \mathcal{B}_{0}\left(t_{\hat{\mathcal{B}}_{0}, t, \varepsilon}\right) .
$$

Thus $\gamma_{T_{\mathcal{B}_{0}, t} \hat{\mathcal{B}}_{0}, t, \varepsilon}\left(\hat{\mathcal{B}_{0}}, t\right)$ is bounded in $W$ and

$$
\alpha_{W}\left(\gamma_{T_{\hat{\mathcal{B}}_{0}, t_{\hat{\mathcal{B}}}, t, \varepsilon}}\left(\hat{\mathcal{B}_{0}}, t\right)\right) \leq \alpha_{W}\left(U\left(t, t_{\hat{\mathcal{B}}_{0}, t, \varepsilon}\right) \mathcal{B}_{0}\left(t_{\hat{\mathcal{B}}_{0}, t, \varepsilon}\right)\right)<\varepsilon
$$

which proves the claim.

\section{Application to reaction-diffusion equations}

Following [17] we consider

$$
\partial_{t} u=\triangle u-f(u)+g(t), x \in \Omega, \quad u(t, x)=0, x \in \partial \Omega, t>s,
$$

$$
u(s, x)=u_{s}(x), x \in \Omega
$$

for a bounded smooth domain $\Omega \subset \mathbb{R}^{N}$ and $s \in \mathbb{R}$.

We assume that $f \in C(\mathbb{R}, \mathbb{R})$ and there exists $p \geq 2$ and positive constants $C_{i}, i=1, \ldots, 4$ such that

$$
C_{1}|y|^{p}-C_{2} \leq f(y) y \leq C_{3}|y|^{p}+C_{4}, y \in \mathbb{R} .
$$

We also require that there exists $C_{5}>0$ such that

$$
f \in C^{1}(\mathbb{R}, \mathbb{R}) \text { and } f^{\prime}(y) \geq-C_{5}, y \in \mathbb{R} .
$$

Moreover, we assume that

$$
g \in L_{l o c}^{2}\left(\mathbb{R} ; L^{2}(\Omega)\right)
$$

and $u_{s} \in L^{2}(\Omega)$. 
3.1. Weak solutions and the process. In order to formulate the definition of a weak solution to the above problem, we take into account the growth condition (3.3) and fix $r \geq 1$ such that $H_{0}^{r}(\Omega) \subset L^{p}(\Omega)$; in fact, we take

$$
r \geq \max \left\{N \frac{p-2}{2 p}, 1\right\} \text { for } p>2 \quad \text { and } \quad r=1 \text { for } p=2 .
$$

Definition 3.1. A function $u:[s, T] \times \Omega \rightarrow \mathbb{R}$ is called a weak solution of (3.1) if

$$
u \in L^{2}\left(s, T ; H_{0}^{1}(\Omega)\right) \cap L^{p}\left(s, T ; L^{p}(\Omega)\right)
$$

and satisfies the equation

$$
\partial_{t} u=\triangle_{D} u-f(u)+g
$$

in the space $L^{p^{*}}\left(s, T ; H^{-r}(\Omega)\right)$, where $r$ is set in (3.6), $\partial_{t} u$ denotes a distributional derivative and $\triangle_{D}: H_{0}^{1}(\Omega) \rightarrow H^{-1}(\Omega)$ is the operator given by $\left\langle\triangle_{D} u, v\right\rangle=-(\nabla u, \nabla v)_{L^{2}(\Omega)}, u, v \in H_{0}^{1}(\Omega)$.

It follows from [7, Theorem II.1.8] that every weak solution has a continuous representative $u \in C\left([s, T] ; L^{2}(\Omega)\right)$. Moreover, the Galerkin approximation yields the following result on existence and uniqueness of weak solutions (see [7, Theorems XV.3.1,3.3], [21, Theorem 8.4]).

Theorem 3.2. Under the assumptions (3.3), (3.5) for any $T>s, s, T \in \mathbb{R}$ and $u_{s} \in L^{2}(\Omega)$ the equation (3.1) has a weak solution, i.e.,

$$
u \in L^{2}\left(s, T ; H_{0}^{1}(\Omega)\right) \cap L^{p}\left(s, T ; L^{p}(\Omega)\right)
$$

with $\partial_{t} u \in L^{p^{*}}\left(s, T ; H^{-r}(\Omega)\right)$, where $r \geq 1$ is defined in (3.6) and

$$
\partial_{t} u=\triangle_{D} u-f(u)+g \text { in } L^{p^{*}}\left(s, T ; H^{-r}(\Omega)\right)
$$

and $u(s)=u_{s}$ in $L^{2}(\Omega)$. In particular, we have $u \in C\left([s, T] ; L^{2}(\Omega)\right)$.

If (3.4) also holds, then the solution is unique and for $t \in[s, T]$

$$
\|u(t)-v(t)\|_{L^{2}(\Omega)} \leq e^{C_{5}(t-s)}\left\|u_{s}-v_{s}\right\|_{L^{2}(\Omega)}, u_{s}, v_{s} \in L^{2}(\Omega) .
$$

We define the process

$$
U(t, s) u_{s}=u^{T}(t), t \geq s
$$

where $s \leq t \leq T$ and $u^{T}$ is the weak solution on $[s, T]$ starting from $u_{s}$.

Note that the fact that $u \in C\left([s, T] ; L^{2}(\Omega)\right)$ and (3.8) imply that

$$
\left\{(t, s) \in \mathbb{R}^{2}: t \geq s\right\} \times L^{2}(\Omega) \ni\left(t, s, u_{s}\right) \mapsto U(t, s) u_{s} \in L^{2}(\Omega)
$$

is continuous. Additionally, more regular initial conditions lead to more regular solutions (see [21, Theorem 8.5]). 
Theorem 3.3. Under the assumptions (3.3)-(3.5) for any $T>s, s, T \in \mathbb{R}$ and $u_{s} \in H_{0}^{1}(\Omega) \cap L^{p}(\Omega)$ the equation (3.1) has a unique solution

$$
u \in L^{2}\left(s, T ; H^{2}(\Omega) \cap H_{0}^{1}(\Omega)\right) \cap L^{\infty}\left(s, T ; H_{0}^{1}(\Omega) \cap L^{p}(\Omega)\right)
$$

with $\partial_{t} u \in L^{2}\left(s, T ; L^{2}(\Omega)\right)$ such that

$$
\partial_{t} u=\triangle_{D} u-f(u)+g \text { in } L^{2}\left(s, T ; L^{2}(\Omega)\right)
$$

and $u(s)=u_{s}$ in $H_{0}^{1}(\Omega) \cap L^{p}(\Omega)$. In particular, $u(t) \in H_{0}^{1}(\Omega) \cap L^{p}(\Omega)$ for every $t \in[s, T]$ and

$$
u \in C\left([s, T] ; H_{0}^{1}(\Omega)\right) \cap C_{w}\left([s, T] ; H_{0}^{1}(\Omega) \cap L^{p}(\Omega)\right)
$$

and the function

$$
[s, T] \ni t \mapsto\||\nabla u(t)|\|_{L^{2}(\Omega)}^{2} \in \mathbb{R}
$$

is absolutely continuous with

$$
\partial_{t}\||\nabla u(t)|\|_{L^{2}(\Omega)}^{2}=-2\left(\partial_{t} u(t), \triangle_{D} u(t)\right)_{L^{2}(\Omega)} \text { for a.e. } t \in[s, T] .
$$

3.2. The process is $\left(L^{2}(\Omega)-H_{0}^{1}(\Omega)\right)$ closed. Since

$$
u \in C\left([s, T] ; L^{2}(\Omega)\right) \cap C\left((s, T] ; H_{0}^{1}(\Omega)\right) \cap C_{w}\left((s, T] ; H_{0}^{1}(\Omega) \cap L^{p}(\Omega)\right),
$$

if $u_{s} \in L^{2}(\Omega)$, then $u(t) \in H_{0}^{1}(\Omega)$ for every $t>s$ and the continuity of the process $U(t, s): L^{2}(\Omega) \rightarrow L^{2}(\Omega)$ implies its $\left(L^{2}(\Omega)-H_{0}^{1}(\Omega)\right)$ closedness.

3.3. Differential inequalities a.e. in $(s, T)$. Taking the inner product of the equation (3.9) in $L^{2}(\Omega)$ with $u(t)$ we obtain

$$
\partial_{t}\|u(t)\|_{L^{2}(\Omega)}^{2}+\||\nabla u(t)|\|_{L^{2}(\Omega)}^{2} \leq 2 C_{2}|\Omega|+\lambda_{1}^{-1}\|g(t)\|_{L^{2}(\Omega)}^{2} .
$$

Applying Poincaré inequality to (3.10) gives

$$
\partial_{t}\|u(t)\|_{L^{2}(\Omega)}^{2}+\lambda_{1}\|u(t)\|_{L^{2}(\Omega)}^{2} \leq 2 C_{2}|\Omega|+\lambda_{1}^{-1}\|g(t)\|_{L^{2}(\Omega)}^{2} .
$$

Furthermore, taking the inner product of $(3.9)$ in $L^{2}(\Omega)$ with $-\triangle_{D} u(t)$ yields

$$
\begin{aligned}
\partial_{t}\||\nabla u(t)|\|_{L^{2}(\Omega)}^{2}+\lambda_{1} & \||\nabla u(t)|\|_{L^{2}(\Omega)}^{2} \\
& \leq 2 C_{5}\||\nabla u(t)|\|_{L^{2}(\Omega)}^{2}+\|g(t)-f(0)\|_{L^{2}(\Omega)}^{2} .
\end{aligned}
$$

Let $P_{n}$ denote the projection of $H_{0}^{1}(\Omega)$ on the space spanned by $n$ first eigenfunctions of $-\triangle_{D}$. We also set $Q_{n}=I-P_{n}$, take the inner product 
of the equation (3.9) in $L^{2}(\Omega)$ with $-\triangle_{D} Q_{n} u(t)$ and use properties of the eigenvalues $\lambda_{1} \leq \lambda_{2} \leq \ldots$ to obtain

$$
\begin{aligned}
\partial_{t}\left\|\left|\nabla Q_{n} u(t)\right|\right\|_{L^{2}(\Omega)}^{2}+\lambda_{n+1} & \left\|\left|\nabla Q_{n} u(t)\right|\right\|_{L^{2}(\Omega)}^{2} \\
& \leq 2\left(\|g(t)\|_{L^{2}(\Omega)}^{2}+\|f(u(t))\|_{L^{2}(\Omega)}^{2} .\right.
\end{aligned}
$$

If $N=1,2$ and $2 \leq p<\infty$ or $N \geq 3$ and $2 \leq p \leq 1+\frac{N}{N-2}$, then

$$
\begin{gathered}
\partial_{t}\left\|\left|\nabla Q_{n} u(t)\right|\right\|_{L^{2}(\Omega)}^{2}+\lambda_{n+1}\left\|\left|\nabla Q_{n} u(t)\right|\right\|_{L^{2}(\Omega)}^{2} \leq \\
\leq 2\|g(t)\|_{L^{2}(\Omega)}^{2}+2 C_{6}\left(1+\||\nabla u(t)|\|_{L^{2}(\Omega)}^{2(p-1)}\right),
\end{gathered}
$$

since we have $H_{0}^{1}(\Omega) \subset L^{2(p-1)}(\Omega)$ and

$$
\|f(u)\|_{L^{2}(\Omega)}^{2} \leq C_{6}\left(1+\||\nabla u|\|_{L^{2}(\Omega)}^{2(p-1)}\right) .
$$

3.4. The universe $\mathcal{D}$ and the assumption on $g$. We define the universe of families of nonempty subsets of $L^{2}(\Omega)$

$$
\begin{aligned}
\mathcal{D}=\left\{\hat{D}=\left\{D(t) \subset L^{2}(\Omega):\right.\right. & t \in \mathbb{R}\}: \\
& \left.\lim _{s \rightarrow-\infty} e^{\lambda_{1} s} \sup \left\{\|u\|_{L^{2}(\Omega)}^{2}: u \in D(s)\right\}=0\right\} .
\end{aligned}
$$

So far we have assumed about $g$ that it satisfies (3.5). Now we assume further that

$$
\int_{-\infty}^{t} e^{\lambda_{1} \tau}\|g(\tau)\|_{L^{2}(\Omega)}^{2} d \tau<\infty, t \in \mathbb{R}
$$

3.5. Pullback $\mathcal{D}$-dissipativity. We fix $\hat{D} \in \mathcal{D}$ and $t \in \mathbb{R}$. By definition of $\mathcal{D}$ let $T_{\hat{D}, t}<t-1<t$ be such that for any $s \leq T_{\hat{D}, t}$ and $u_{s} \in D(s)$ we have

$$
e^{\lambda_{1} s}\left\|u_{s}\right\|_{L^{2}(\Omega)}^{2} \leq 2 C_{2} \lambda_{1}^{-1}|\Omega| e^{\lambda_{1}(t-1)} .
$$

We use this and apply Gronwall inequality to (3.11) on $[s, t-1]$ and get

$$
\|u(t-1)\|_{L^{2}(\Omega)}^{2} \leq 4 C_{2}|\Omega| \lambda_{1}^{-1}+\lambda_{1}^{-1} e^{-\lambda_{1}(t-1)} \int_{s}^{t-1} e^{\lambda_{1} \tau}\|g(\tau)\|_{L^{2}(\Omega)}^{2} d \tau .
$$

We integrate (3.10) on the interval $[t-1, t]$ and use (3.18) to obtain

$$
\int_{t-1}^{t}\|u(\tau)\|_{H_{0}^{1}(\Omega)}^{2} d \tau \leq c_{1}\left(1+e^{-\lambda_{1}(t-1)} \int_{-\infty}^{t} e^{\lambda_{1} \tau}\|g(\tau)\|_{L^{2}(\Omega)}^{2} d \tau\right) .
$$


We now apply the uniform Gronwall inequality (see [17, Lemma 3.3]) to (3.12) on $[t-1, t]$ and get

$$
\begin{aligned}
\|u(t)\|_{H_{0}^{1}(\Omega)}^{2} & \leq 2 e^{-\frac{\lambda_{1}}{2}} \int_{t-1}^{t}\|u(\tau)\|_{H_{0}^{1}(\Omega)}^{2} d \tau+ \\
& +e^{-\lambda_{1} t} \int_{t-1}^{t} e^{\lambda_{1} \tau}\left(2 C_{5}\|u(\tau)\|_{H_{0}^{1}(\Omega)}^{2}+\|g(\tau)-f(0)\|_{L^{2}(\Omega)}^{2}\right) d \tau .
\end{aligned}
$$

Inserting (3.19) we obtain

$$
\|u(t)\|_{H_{0}^{1}(\Omega)}^{2} \leq c_{2}\left(1+e^{-\lambda_{1} t+\lambda_{1}} \int_{-\infty}^{t} e^{\lambda_{1} \tau}\|g(\tau)\|_{L^{2}(\Omega)}^{2} d \tau\right)=: r_{0}(t)^{2},
$$

where $c_{2}=c_{2}\left(|\Omega|, C_{2}, C_{5}, \lambda_{1},\|f(0)\|_{L^{2}(\Omega)}^{2}\right)>0$.

We have shown that for any $\hat{D} \in \mathcal{D}$ and $t \in \mathbb{R}$ there exists $T_{\hat{D}, t}<t$ such that

$$
\bigcup_{s \leq T_{\hat{D}, t}} U(t, s) D(s) \subset \bar{B}^{H_{0}^{1}(\Omega)}\left(0, r_{0}(t)\right)=: B_{0}(t) .
$$

We also see that $\hat{B}_{0}=\left\{B_{0}(t): t \in \mathbb{R}\right\} \in \mathcal{D}$. Note that from (3.19) we have

$$
\int_{t-1}^{t}\|u(\tau)\|_{H_{0}^{1}(\Omega)}^{2} d \tau \leq \frac{c_{1}}{c_{2}} r_{0}(t)^{2} .
$$

3.6. $\left(L^{2}(\Omega)-H_{0}^{1}(\Omega)\right)$ pullback $\mathcal{D}$-asymptotic compactness. Below we will verify the $\left(L^{2}(\Omega)-H_{0}^{1}(\Omega)\right)$ pullback $\mathcal{D}$-flattening condition. We fix $\hat{D} \in \mathcal{D}$ and $t \in \mathbb{R}$. Let $s_{0}=s_{0}(\hat{D}, t)<t-2<t$ be such that for any $s \leq s_{0}$ and $u_{s} \in D(s)$ we have

$$
e^{\lambda_{1} s}\left\|u_{s}\right\|_{L^{2}(\Omega)}^{2} \leq 2 C_{2} \lambda_{1}^{-1}|\Omega| e^{\lambda_{1}(t-2)} .
$$

We consider $\sigma \in[t-1, t]$ and have $s \leq s_{0}<t-2 \leq \sigma-1 \leq t-1 \leq \sigma$. We can take $\sigma$ is the role of $t$ in the previous calculations and obtain

$$
\begin{gathered}
\|u(\sigma)\|_{H_{0}^{1}(\Omega)}^{2} \leq c_{2}\left(1+e^{-\lambda_{1} \sigma+\lambda_{1}} \int_{-\infty}^{\sigma} e^{\lambda_{1} \tau}\|g(\tau)\|_{L^{2}(\Omega)}^{2} d \tau\right) \leq \\
\leq c_{2} e^{\lambda_{1}}\left(1+e^{-\lambda_{1} t+\lambda_{1}} \int_{-\infty}^{t} e^{\lambda_{1} \tau}\|g(\tau)\|_{L^{2}(\Omega)}^{2} d \tau\right) .
\end{gathered}
$$

Comparing this with (3.21) we have

$$
\|u(\sigma)\|_{H_{0}^{1}(\Omega)}^{2} \leq e^{\lambda_{1}} r_{0}(t)^{2}, \sigma \in[t-1, t] .
$$

São Paulo J.Math.Sci. 6, 2 (2012), 227-246 
Now we use (3.14) if $N=1,2,2 \leq p<\infty$ or $N \geq 3,2 \leq p \leq 1+\frac{N}{N-2}$. This is the only place where we use this restriction. We apply the uniform Gronwall inequality (see [17, Lemma 3.3]) on $[t-1, t]$ and get

$$
\begin{aligned}
\left\|Q_{n} u(t)\right\|_{H_{0}^{1}(\Omega)}^{2} \leq 2 e^{-\frac{\lambda_{n}+1}{2}} \int_{t-1}^{t}\left\|Q_{n} u(\tau)\right\|_{H_{0}^{1}(\Omega)}^{2} d \tau & \\
& +2 C_{6} e^{-\lambda_{n+1} t} \int_{t-1}^{t} e^{\lambda_{n+1} \tau}\left(1+\|u(\tau)\|_{H_{0}^{1}(\Omega)}^{2 p-2}\right) d \tau \\
& \quad+2 e^{-\lambda_{n+1} t} \int_{t-1}^{t} e^{\lambda_{n+1} \tau}\|g(\tau)\|_{L^{2}(\Omega)}^{2} d \tau .
\end{aligned}
$$

Since $Q_{n}$ is a projection on $H_{0}^{1}(\Omega)$ and (3.22) holds, we get

$$
2 e^{-\frac{\lambda_{n+1}}{2}} \int_{t-1}^{t}\left\|Q_{n} u(\tau)\right\|_{H_{0}^{1}(\Omega)}^{2} d \tau \leq 2 e^{-\frac{\lambda_{n+1}}{2}} \frac{c_{1}}{c_{2}} r_{0}(t)^{2} .
$$

Using (3.24) we also have

$$
\begin{aligned}
2 C_{6} e^{-\lambda_{n+1} t} \int_{t-1}^{t} e^{\lambda_{n+1} \tau} & \left(1+\|u(\tau)\|_{H_{0}^{1}(\Omega)}^{2 p-2}\right) d \tau \\
\leq & 2 C_{6} \lambda_{n+1}^{-1}\left(1+e^{\lambda_{1}(p-1)} r_{0}(t)^{2 p-2}\right) .
\end{aligned}
$$

Concluding we obtain

$$
\begin{array}{r}
\left\|Q_{n} u(t)\right\|_{H_{0}^{1}(\Omega)}^{2} \leq 2 e^{-\frac{\lambda_{n+1}}{2}} \frac{c_{1}}{c_{2}} r_{0}(t)^{2}+2 C_{6} \lambda_{n+1}^{-1}\left(1+e^{\lambda_{1}(p-1)} r_{0}(t)^{2 p-2}\right)+ \\
+2 e^{-\lambda_{n+1} t} \int_{t-1}^{t} e^{\lambda_{n+1} \tau}\|g(\tau)\|_{L^{2}(\Omega)}^{2} d \tau=A_{n}(t)+B_{n}(t)+C_{n}(t) .
\end{array}
$$

Note that $A_{n}(t) \rightarrow 0$ and $B_{n}(t) \rightarrow 0$ as $n \rightarrow \infty$ uniformly w.r.t. $u_{s} \in D(s)$.

To show that the same holds for $C_{n}(t)$, we fix $\varepsilon>0$ and choose $0<\delta<1$ such that

$$
2 \int_{t-\delta}^{t}\|g(\tau)\|_{L^{2}(\Omega)}^{2} d \tau<\frac{\varepsilon}{2}
$$

We also choose $n_{0}=n_{0}(\varepsilon, t) \in \mathbb{N}$ such that for $n \geq n_{0}$

$$
2 e^{-\delta \lambda_{n+1}} e^{-\lambda_{1}(t-\delta)} \int_{-\infty}^{t} e^{\lambda_{1} \tau}\|g(\tau)\|_{L^{2}(\Omega)}^{2} d \tau<\frac{\varepsilon}{2}
$$

These and the fact that $\lambda_{n+1} \geq \lambda_{1}$ imply

$$
C_{n}(t)=2 e^{-\lambda_{n+1} t}\left(\int_{t-1}^{t-\delta}+\int_{t-\delta}^{t}\right) e^{\lambda_{n+1} \tau}\|g(\tau)\|_{L^{2}(\Omega)}^{2} d \tau \leq
$$




$$
\leq 2 e^{-\lambda_{n+1} t} \int_{t-1}^{t-\delta} e^{\left(\lambda_{n+1}-\lambda_{1}\right) \tau} e^{\lambda_{1} \tau}\|g(\tau)\|_{L^{2}(\Omega)}^{2} d \tau+2 \int_{t-\delta}^{t}\|g(\tau)\|_{L^{2}(\Omega)}^{2} d \tau<\varepsilon,
$$

which shows that $C_{n}(t) \rightarrow 0$ as $n \rightarrow \infty$ uniformly w.r.t. $u_{s} \in D(s)$.

We have shown that for any $\hat{D} \in \mathcal{D}$ and $t \in \mathbb{R}$

$$
\exists_{s_{0}=s_{0}(\hat{D}, t)<t} \forall_{\varepsilon>0} \exists_{n_{0} \in \mathbb{N}} \forall_{n \geq n_{0}} \forall_{s \leq s_{0}} \forall_{u_{s} \in D(s)}\left\|Q_{n} U(t, s) u_{s}\right\|_{H_{0}^{1}(\Omega)}<\varepsilon .
$$

Hence there exists $t_{0}=t_{0}(\hat{D}, t) \leq \min \left\{T_{\hat{D}, t}, s_{0}(\hat{D}, t)\right\}$ such that

$$
\forall_{\varepsilon>0} \exists_{n_{0} \in \mathbb{N}} \forall_{n \geq n_{0}}\left(I-P_{n}\right)\left(\gamma_{t_{0}}(\hat{D}, t)\right) \subset B^{H_{0}^{1}(\Omega)}(0, \varepsilon) .
$$

Recall that $P_{n}: H_{0}^{1}(\Omega) \rightarrow V_{n}$ is a projector, where $V_{n}$ is a finite dimensional subspace of $H_{0}^{1}(\Omega)$ and $\gamma_{t_{0}}(\hat{D}, t) \subset B_{0}(t)$, which is a bounded subset of $H_{0}^{1}(\Omega)$. Hence

$$
P_{n}\left(\gamma_{t_{0}}(\hat{D}, t)\right) \text { is bounded in } H_{0}^{1}(\Omega) .
$$

3.7. $\left(L^{2}(\Omega)-H_{0}^{1}(\Omega)\right)$ pullback $\mathcal{D}$-attractor. We apply Corollary 2.17 and obtain the existence of the $\left(L^{2}(\Omega)-H_{0}^{1}(\Omega)\right)$ pullback $\mathcal{D}$-attractor.

Theorem 3.4. Let $N=1,2,2 \leq p<\infty$ or $N \geq 3,2 \leq p \leq 1+$ $\frac{N}{N-2}$. Under the assumptions (3.3), (3.4) on $f$ and for $g \in L_{\text {loc }}^{2}\left(\mathbb{R} ; L^{2}(\Omega)\right)$ satisfying

$$
\int_{-\infty}^{t} e^{\lambda_{1} \tau}\|g(\tau)\|_{L^{2}(\Omega)}^{2} d \tau<\infty, t \in \mathbb{R}
$$

the process for the problem (3.1), (3.2) possesses $\left(L^{2}(\Omega)-H_{0}^{1}(\Omega)\right)$ pullback $\mathcal{D}$-attractor with the universe

$$
\begin{aligned}
\mathcal{D}=\left\{\hat{D}=\left\{D(t) \subset L^{2}(\Omega):\right.\right. & t \in \mathbb{R}\}: \\
& \left.\lim _{s \rightarrow-\infty} e^{\lambda_{1} s} \sup \left\{\|u\|_{L^{2}(\Omega)}^{2}: u \in D(s)\right\}=0\right\} .
\end{aligned}
$$

It is given by

$$
\mathcal{A}(t)=\omega_{H_{0}^{1}(\Omega)}\left(\hat{\mathcal{B}_{0}}, t\right), t \in \mathbb{R}
$$

\section{References}

[1] J. M. Arrieta, J. W. Cholewa, T. Dlotko, A. Rodríguez-Bernal, Dissipative parabolic equations in locally uniform spaces, Math. Nachr. 280 (2007), 1643-1663.

[2] A. V. Babin, M. I. Vishik, Attractors of Evolution Equations, North-Holland, Amsterdam, 1992.

[3] T. Caraballo, A. N. Carvalho, J. A. Langa, F. Rivero, Existence of pullback attractors for pullback asymptotically compact processes, Nonlinear Anal. 72 (2010), 1967-1976. 
[4] T. Caraballo, G. Łukaszewicz, J. Real, Pullback attractors for asymptotically compact non-autonomous dynamical systems, Nonlinear Anal. 64 (2006), 484498.

[5] A. N. Carvalho, J. A. Langa, J. C. Robinson, Attractors for Infinite-Dimensional Non-Autonomous Dynamical Systems, Applied Math. Sciences 182, Springer, 2012.

[6] D. N. Cheban, Global Attractors of Non-Autonomous Dissipative Dynamical Systems, World Scientific Publishing Co., 2004.

[7] V. Chepyzhov, M. Vishik, Attractors for Equations of Mathematical Physics, Amer. Math. Soc., Providence, RI, 2002.

[8] J. W. Cholewa, R. Czaja, G. Mola, Remarks on the fractal dimension of bi-space global and exponential attractors, Boll. Unione Mat. Ital. (9) 1 (2008), 121-145.

[9] J. W. Cholewa, T. Dlotko, Global Attractors in Abstract Parabolic Problems, Cambridge University Press, Cambridge, 2000.

[10] J. W. Cholewa, A. Rodríguez-Bernal, Extremal equilibria for monotone semigroups in ordered spaces with application to evolutionary equations, J. Differential Equations 249 (2010), 485-525.

[11] H. Crauel, A. Debussche, F. Flandoli, Random attractors, J. Dynam. Differential Equations 9 (1997), 307-341.

[12] M. M. Day, Reflexive Banach spaces not isomorphic to uniformly convex spaces, Bull. Amer. Math. Soc. 47 (1941), 313-317.

[13] J. García-Luengo, P. Marín-Rubio, J. Real, Pullback attractors in $V$ for nonautonomous 2D-Navier-Stokes equations and their tempered behaviour, J. Differential Equations 252 (2012), 4333-4356.

[14] J. K. Hale, Asymptotic Behavior of Dissipative Systems, Amer. Math. Soc., Providence, RI, 1988.

[15] P. E. Kloeden, M. Rasmussen, Nonautonomous Dynamical Systems, Mathematical Surveys and Monographs 176, Amer. Math. Soc., Providence, RI, 2011.

[16] Y. Li, C. Zhong, Pullback attractors for the norm-to-weak continuous process and application to the nonautonomous reaction-diffusion equations, Appl. Math. Comput. 190 (2007), 1020-1029.

[17] G. Eukaszewicz, On pullback attractors in $H_{0}^{1}$ for nonautonomous reactiondiffusion equations, Internat. J. Bifur. Chaos Appl. Sci. Engrg. 20 (2010), 26372644.

[18] Q. Ma, S. Wang, C. Zhong, Necessary and sufficient conditions for the existence of global attractors for semigroups and applications, Indiana Univ. Math. J. 51 (2002), 1541-1570.

[19] P. Marín-Rubio, J. Real, On the relation between two different concepts of pullback attractors for non-autonomous dynamical systems, Nonlinear Anal. 71 (2009), 3956-3963.

[20] V. Pata, S. Zelik, A result on the existence of global attractors for semigroups of closed operators, Commun. Pure Appl. Anal. 6 (2007), 481-486.

[21] J. C. Robinson, Infinite-Dimensional Dynamical Systems - An Introduction to Dissipative Parabolic PDEs and the Theory of Global Attractors, Cambridge Univerisity Press, Cambridge, 2001.

[22] G. R. Sell, Y. You, Dynamics of Evolutionary Equations, Springer, 2002.

[23] I. Singer, Best Approximation in Normed Linear Spaces by Elements of Linear Subspaces, Springer-Verlag, Berlin, 1970.

[24] I. Singer, The Theory of Best Approximation and Functional Analysis, Society of Industrial and Applied Mathematics, Philadelphia, Pennsylvania, 1974. 
[25] T. Tachim Medjo, Pullback attractors for closed cocycles, Nonlinear Anal. 73 (2010), 2737-2751.

[26] R. Temam, Infinite-Dimensional Dynamical Systems in Mechanics and Physics, Springer, New York, 1988.

[27] Y. Wang, C. Zhong, S. Zhou, Pullback attractors of nonautonomous dynamical systems, Discrete Contin. Dyn. Syst. 16 (2006), 587-614.

[28] L. Yang, Uniform attractors for the closed process and application to the reaction-diffusion equation with dynamical boundary condition, Nonlinear Anal. 71 (2009), 4012-4025.

[29] C. Zhong, M. Yang, C. Sun, The existence of global attractors for the norm-toweak continuous semigroup and application to the nonlinear reaction-diffusion equations, J. Differential Equations 223 (2006), 367-399. 